\title{
Interacting lattice electrons with disorder in two dimensions: Numerical evidence for a metal-insulator transition with a universal critical conductivity
}

\author{
Prabuddha B. Chakraborty, ${ }^{1, *}$ Krzysztof Byczuk, ${ }^{2}$ and Dieter Vollhardt ${ }^{1}$ \\ ${ }^{1}$ Theoretical Physics III, Center for Electronic Correlations and Magnetism, Institute of Physics, University of Augsburg, \\ D-86135, Augsburg, Germany \\ ${ }^{2}$ Faculty of Physics, University of Warsaw, ul. Hoża 69, PL-00-681, Warsaw, Poland
}

(Received 8 July 2011; published 25 July 2011)

\begin{abstract}
The dc conductivity of electrons on a square lattice interacting with a local repulsion in the presence of disorder is computed by means of quantum Monte Carlo simulations. We provide evidence for the existence of a transition from an Anderson insulator to a correlated disordered metal with a universal value of the critical dc conductivity $\sigma_{\mathrm{dc}, \text { crit }}=(1.19 \pm 0.02) e^{2} / h$ at the transition.
\end{abstract}

DOI: 10.1103/PhysRevB.84.035121

PACS number(s): 71.10.Fd, 71.27.+a, 71.30.+h

\section{INTRODUCTION}

The Coulomb interaction between the electrons and the presence of disorder both strongly affect the properties of solids. ${ }^{1-5}$ Namely, electronic correlations and randomness are separate driving forces behind metal-insulator transitions (MITs) due to the localization and delocalization of particles. While the electronic repulsion may lead to a Mott-Hubbard MIT, ${ }^{6}$ the scattering of noninteracting particles from randomly distributed impurities can cause Anderson localization. ${ }^{7,8}$ The simultaneous presence of disorder and interactions lead to further subtle many-body effects, which raise fundamental questions in theory and experiment not only in solid state physics, ${ }^{1-6,8-12}$ but also in the field of cold atoms in optical lattices. ${ }^{13}$

According to the scaling theory of Anderson localization $^{14,15}$ noninteracting electrons in two spatial dimensions $(d=2)$ are localized in the presence of disorder. Hence, in the thermodynamic limit at zero temperature, there is no metallic state in $d=2$. By contrast, the experimental observation of an MIT in resistivity measurements on various high-mobility heterostructure samples and Si-metal-oxide-semiconductor field-effect transistors (Si-MOSFETs) ${ }^{16}$ clearly indicates that interactions can turn an Anderson insulator into a metal. Near-perfect scaling of the resistivity data ${ }^{17}$ was taken as evidence for the presence of a quantum critical point ${ }^{18}$ between the metallic and the Anderson localized state. ${ }^{4,5}$ Recent investigations ${ }^{19,20}$ based on a nonlinear sigma model $(\mathrm{NL} \sigma \mathrm{M})$ for interacting electrons with disorder in the continuum confirm the existence of such a quantum critical point, which is characterized by a universal value of the dc resistivity. Universal critical conductivities were also discussed in other two-dimensional systems; for example, in connection with the transition from a superconductor (superfluid) to an insulator, ${ }^{21,22}$ in the integer quantum Hall effect, ${ }^{23}$ and in graphene. ${ }^{24}$

Numerical investigations of the interplay between disorder and interactions usually address electrons on a lattice rather than in the continuum. Various approaches include HartreeFock investigations in three ${ }^{25}$ and two dimensions ${ }^{26}$ quantum Monte Carlo (QMC) simulations, ${ }^{27-29}$ and dynamical meanfield theory. ${ }^{30-33}$ In their QMC studies of two-dimensional lattice electrons, Denteneer et al. ${ }^{27,28}$ indeed found a phase transition between an Anderson insulator and a metallic phase, in accordance with experiment. ${ }^{5}$ There has also been the proposal of the MIT as a percolation transition. ${ }^{34}$

In this paper we provide evidence through extensive QMC simulations that, in the Anderson-Hubbard model in $d=2$, there exists a transition between a metallic phase and an Anderson insulator, and that this transition takes place at a value of the dc conductivity $\sigma_{\mathrm{dc} \text {,crit }}$ which is essentially independent of the critical interaction, critical disorder, and particle density. The computation of such a universal value of the critical dc conductivity provides an explicit link to results obtained from effective theories in the continuum. ${ }^{19}$ Indeed, numerical investigations of microscopic lattice models can provide details of the properties of a system at a quantum critical point that are not accessible within effective perturbative approaches.

\section{MODEL AND METHOD OF INVESTIGATION}

Our investigation of interacting electrons in the presence of disorder is based on the Anderson-Hubbard Hamiltonian on a square lattice

$$
H=T\left\{\epsilon_{i}\right\}+U \sum_{i} n_{i \uparrow} n_{i \downarrow}
$$

Here,

$$
T\left\{\epsilon_{i}\right\}=-t \sum_{\langle i j\rangle \sigma} c_{i \sigma}^{\dagger} c_{j \sigma}+\sum_{i \sigma}\left(\epsilon_{i}-\mu\right) n_{i \sigma},
$$

is the single-electron part where $c_{i \sigma}^{\dagger}\left(c_{i \sigma}\right)$ are fermion creation (annihilation) operators for site $\mathbf{R}_{i}$ and spin $\sigma, n_{i \sigma}=c_{i \sigma}^{\dagger} c_{i \sigma}$ is the operator for the local density, $\mu$ denotes the chemical potential, and $t$ is the hopping amplitude for electrons between nearest-neighbor sites. The local energies $\epsilon_{i}$ are random variables which are sampled uniformly from the interval $[-\Delta / 2, \Delta / 2]$; hence the width $\Delta$ characterizes the strength of the disorder. The interaction is assumed to be repulsive $(U>0)$. The model is solved numerically using determinantal QMC (DQMC), ${ }^{35}$ where the interval $[0, \beta]\left[\beta=1 /\left(k_{B} T\right)\right]$ is partitioned according to $\beta=L \Delta \tau$, with $\Delta \tau$ being the size of a small step in the imaginary time direction, and $L$ being the number of imaginary time slices. The partition function $Z$ is then decomposed according to the SuzukiTrotter formula. ${ }^{36}$ In the next step, a Hubbard-Stratonovich 
transformation is performed whereby the interaction problem is reduced to noninteracting electrons in the presence of fluctuating fields described by Ising variables on every space- (imaginary) time lattice site. ${ }^{37}$ The electrons can then be integrated out. The calculation of quantities such as the Green function, electronic density, and two-particle correlation functions proceeds with Monte Carlo sampling of the various configurations of the Ising degrees of freedom. The hopping integral $t$ sets the unit of energy, and the simulation now contains three independent energy scales: the disorder strength $\Delta$, the interaction strength $U$, and the temperature $T$.

To evaluate the dc conductivity, we compute the electronic current density operator

$$
j_{x}\left(\mathbf{R}_{i}\right)=\frac{i e a t}{\hbar} \sum_{\sigma}\left(c_{i+\mathbf{e}_{x} \sigma}^{\dagger} c_{i \sigma}-c_{i \sigma}^{\dagger} c_{i+\mathbf{e}_{x} \sigma}\right),
$$

where $\mathbf{e}_{x}$ denotes a translation in $x$ direction by a lattice constant $a$. This leads to the time-dependent current density operator

$$
j_{x}\left(\mathbf{R}_{i}, \tau\right)=e^{H \tau / \hbar} j_{x}\left(\mathbf{R}_{i}\right) e^{-H \tau / \hbar},
$$

where $\tau$ is the imaginary (Matsubara) time. The position-space Fourier transform of the current operator, $j_{x}(\mathbf{q}, \tau)$, is then used to calculate the current-current correlation function

$$
\Lambda_{x x}(\mathbf{q}, \tau)=\left\langle j_{x}(\mathbf{q}, \tau) j_{x}(-\mathbf{q}, \tau=0)\right\rangle .
$$

Within linear response theory, the dc conductivity is obtained from

$$
\sigma_{\mathrm{dc}}=\lim _{\omega \rightarrow 0} \frac{\operatorname{Im} \Lambda_{x x}(\mathbf{q}=0, \omega)}{\omega} .
$$

The current-current correlation function in Matsubara time is related to the imaginary part of the current-current correlation function in real frequency through the integral transform

$$
\Lambda_{x x}(\mathbf{q}, \tau)=\int_{-\infty}^{\infty} \frac{d \omega}{\pi} \frac{e^{-\omega \tau}}{1-e^{-\beta \omega}} \operatorname{Im} \Lambda_{x x}(\mathbf{q}, \omega) .
$$

DQMC simulations can compute $\Lambda_{x x}(\mathbf{q}, \tau)$, but to determine $\sigma_{\mathrm{dc}}$ it is necessary to obtain $\operatorname{Im} \Lambda_{x x}(\mathbf{q}, \omega)$. For sufficiently low temperatures, the exponential decay of the bosonic kernel $K(\omega, \tau ; \beta)=\frac{e^{-\omega \tau}}{1-e^{-\beta \omega}}$ for $\tau=\beta / 2$ ensures that the integral contributes only for small $\omega$, where the substitution arising from linear response, Eq. (6), is valid (for a discussion of the accuracy of this approximation see, e.g., Refs. [ 27-29,38]). Replacing $\tau$ by $\beta / 2$ and $\operatorname{Im} \Lambda_{x x}(\mathbf{q}, \omega)$ by $\omega \sigma_{\mathrm{dc}}$, the integral can be carried out analytically and yields the dc conductivity as a function of temperature for different values of the interaction $U$ and disorder strength $\Delta$ :

$$
\sigma_{\mathrm{dc}}=\frac{\beta^{2}}{\pi} \Lambda_{x x}\left(\mathbf{q}=0, \tau=\frac{\beta}{2}\right) .
$$

In the following discussion, we set $t=1$.

There are two sources of statistical error in this analysis: one due to the QMC simulations and the other due to the disorder averaging. For all parameter sets studied here, the intrinsic QMC error for any given disorder realization is much smaller than the error arising from different disorder realizations. These two sources of error are completely independent, and a combined error analysis including both of them cannot be justified. Therefore, the error bars shown are always the larger of the two (i.e., arising from averaging over different disorder realizations). When the error bars are of the order of, or smaller, than the symbols they are not shown. Otherwise, they are shown explicitly.

The conductivity data is averaged over 10 disorder realizations at high temperatures $(T=1,0.5,0.333,0.25)$, up to 80 disorder realizations for intermediate temperatures $(T=$ $0.2,0.167)$, and up to 100 disorder realizations for the two lowest temperatures $(T=0.125,0.1)$. The number of disorder realizations are chosen such that the error bars are comparable across the entire temperature range.

\section{RESULTS}

In Figure 1, the dc conductivity is shown as a function of $\mathrm{T}$ for several values of the disorder strength $\Delta$. Initially, when the value of the disorder strength is less than about $\Delta=10$, the slope of the conductivity curve at low temperatures is negative (i.e., the conductivity decreases with increasing temperature), implying that the system is metallic. As the disorder strength is increased, the low-temperature conductivity develops a positive slope, which is the signature of an insulator. Since the system is far from half-filling, such that a Mott-Hubbard MIT does not occur, these results indicate a transition between a metallic and an Anderson localized state. The dc-conductivity values of Fig. 1 (and all other subsequent figures and tables) have been checked for system-size dependence with $N_{s}=$ $8 \times 8, N_{s}=10 \times 10$, and $N_{s}=12 \times 12$, and the results are unchanged.

On the basis of Fig. 1 neither the critical disorder strength nor the value of the dc conductivity $\sigma_{\mathrm{dc}}$ at the critical point can be determined accurately. In Fig. 2 we therefore plot $\sigma_{\mathrm{dc}}$ as a function of the disorder $\Delta$ for the three lowest

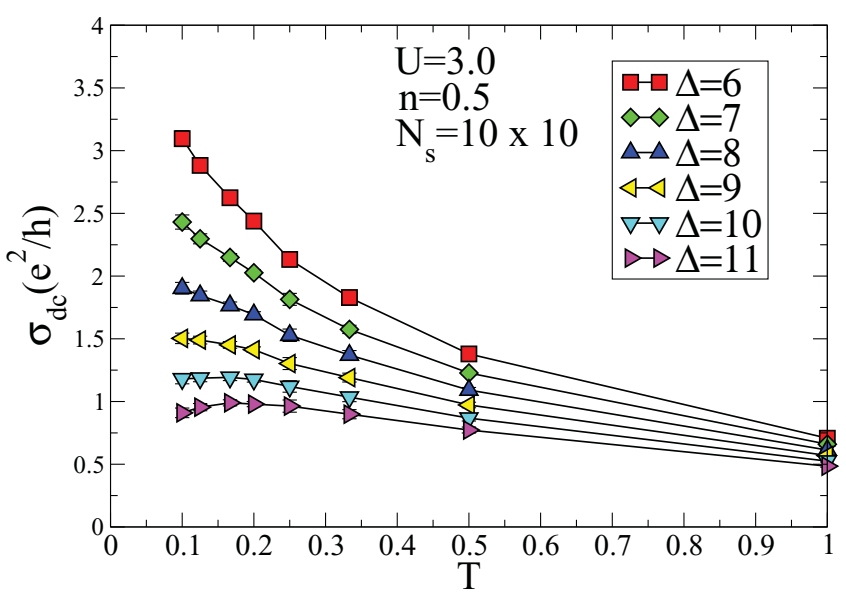

FIG. 1. (Color online) Curves of the dc conductivity vs temperature at electron density $n=0.5$ (quarter filling) and interaction $U=3$ on a $10 \times 10$ square lattice computed for different values of the disorder strength $\Delta$ (see inset). 


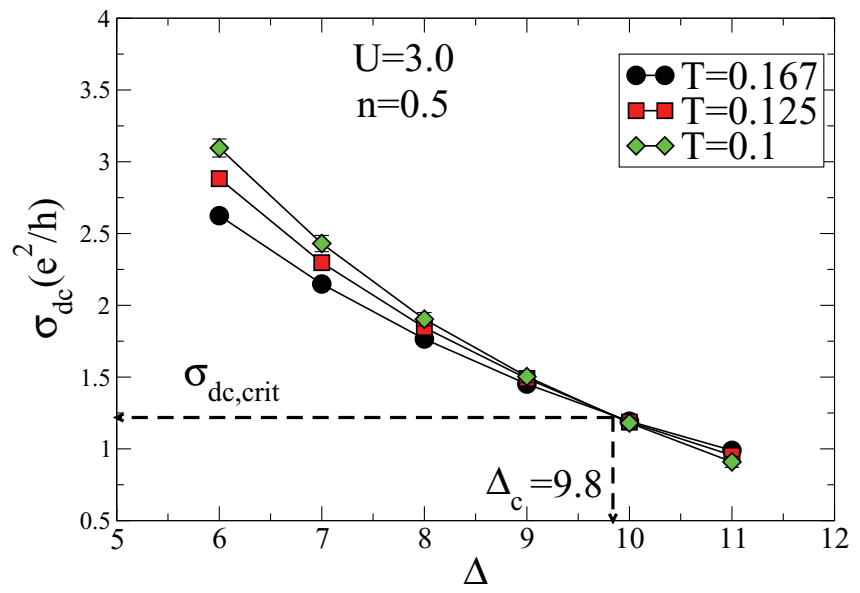

FIG. 2. (Color online) Plot of the critical conductivity $\sigma_{\mathrm{dc}}$ vs disorder strength $\Delta$ for three temperatures and the same values of $U$ and $n$ as in Fig. 1. The well-defined crossing point determines the critical disorder strength as $\Delta_{\mathrm{c}}=9.8$. The conductivity at the critical disorder has the value $\sigma_{\mathrm{dc}, \text { crit }}=1.19 e^{2} / h$.

temperatures simulated here, (i.e., $T=0.167,0.125,0.1$ ). When $\Delta<\Delta_{\mathrm{c}}$, the dc conductivity increases with decreasing temperature (metallic behavior), while for $\Delta>\Delta_{\mathrm{c}}$, the conductivity decreases with decreasing temperature (insulating behavior). The three curves shown in Fig. 2 display a well-defined crossing point, at which the dc conductivity is independent of temperature, thereby marking the critical point for the MIT. From the location of the crossing point one can read off the value of the critical disorder strength $\Delta_{\mathrm{c}}$. For $U=3.0$ at quarter-filling $(n=0.5)$ we find $\Delta_{\mathrm{c}}=9.8$, while the value of the critical conductivity is $\sigma_{\mathrm{dc}, \mathrm{crit}}=1.19 e^{2} / h$.

In Figs. 3 and 4 we show the same plots for a different parameter set $(U=1.0, n=0.3)$. The values of the dc conductivity for similar strengths of disorder are seen to be very different for the two parameter sets. For

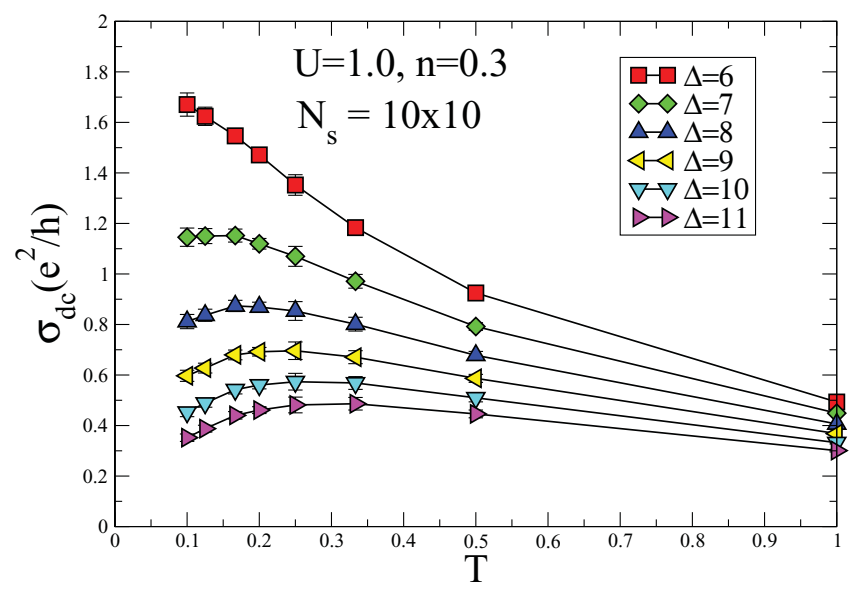

FIG. 3. (Color online) Curves of dc conductivity vs temperature at electron density $n=0.3$ and interaction $U=1$ on a $10 \times 10$ square lattice computed for different values of the disorder strength $\Delta$ (see inset).

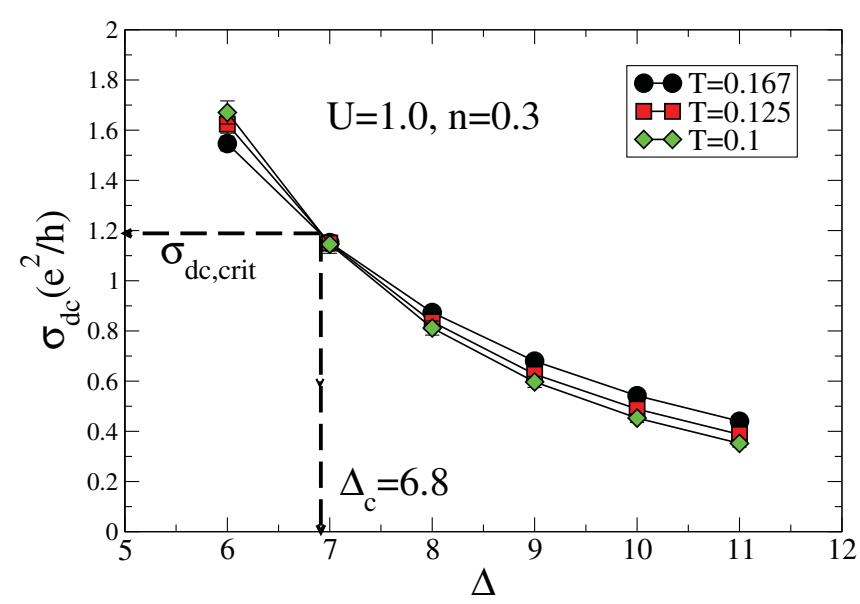

FIG. 4. (Color online) Plot of the critical conductivity $\sigma_{\mathrm{dc}}$ vs disorder strength $\Delta$ for three temperatures and the same values of $U$ and $n$ as in Fig. 3. The well-defined crossing point determines the critical disorder strength as $\Delta_{\mathrm{c}}=6.8$. The conductivity at the critical disorder has the value $\sigma_{\mathrm{dc}, \text { crit }}=1.19 e^{2} / h$.

example, for $\Delta=6$ at $T=0.1$, where metallic behavior is found for both parameter sets, $\sigma_{\mathrm{dc}}=3.1 e^{2} / h$ (cf. Fig. 1) for $(U=3.0, n=0.5)$, and $\sigma_{\mathrm{dc}}=1.67 e^{2} / h$ (cf. Fig. 3) for ( $U=1.0, n=0.3$ ). Similarly, for $\Delta=11$ at $T=0.1$, when both systems are insulating, $\sigma_{\mathrm{dc}}=0.91 e^{2} / h$ (cf. Fig. 1) for $(U=3.0, n=0.5)$, and $\sigma_{\mathrm{dc}}=0.35 e^{2} / h$ (cf. Fig. 3) for $(U=$ $1.0, n=0.3)$.

Using the crossing diagram in Fig. 4 we can again determine the strength of the critical disorder and the critical dc conductivity and find $\Delta_{\mathrm{c}}=6.8$ and $\sigma_{\mathrm{dc} \text {,crit }}=1.19 e^{2} / \mathrm{h}$. Comparing the results of the two parameter sets $(U=3.0, n=0.5)$ and ( $U=1.0, n=0.3$ ), we find that, although the values of the dc conductivity in the metallic and insulating regimes as well as the critical disorder strength $\Delta_{c}$ depend strongly on the parameter set, the critical dc conductivity $\sigma_{\mathrm{dc} \text {,crit }}$ is apparently independent of the values of the microscopic parameters $U$ and $n$.

TABLE I. The eight parameter sets of the interaction strength $U$ and electron density $n$ employed in our investigation of correlated electrons in the presence of disorder on a square lattice are listed together with the computed critical disorder strength $\Delta_{c}$ and the critical dc conductivity $\sigma_{\mathrm{dc}, \text { crit }}$ at the transition between a disordered metal and an Anderson insulator. All simulations were carried out on a $10 \times 10$ square lattice.

\begin{tabular}{lcccc}
\hline \hline Index & $U$ & $n$ & $\Delta_{\mathrm{c}}$ & $\sigma_{\mathrm{dc}, \text { crit }}\left(e^{2} / h\right)$ \\
\hline$a$ & 1.0 & 0.3 & 6.8 & 1.19 \\
$b$ & 1.0 & 0.5 & 6.9 & 1.21 \\
$c$ & 1.0 & 0.6 & 6.9 & 1.25 \\
$d$ & 2.0 & 0.3 & 7.8 & 1.07 \\
$e$ & 2.0 & 0.5 & 7.9 & 1.26 \\
$f$ & 3.0 & 0.3 & 8.6 & 1.19 \\
$g$ & 3.0 & 0.5 & 9.8 & 1.19 \\
$h$ & 3.0 & 0.6 & 10.5 & 1.19 \\
\hline \hline
\end{tabular}


TABLE II. Relative spread of the four quantities $U, n, \Delta_{c}$, and $\sigma_{\mathrm{dc}, \text { crit }}$ (in percent change, see text).

\begin{tabular}{lrccr}
\hline \hline Index & $\delta U$ & $\delta n$ & $\delta \Delta_{\mathrm{c}}$ & $\delta \sigma_{\mathrm{dc}, \text { crit }}$ \\
\hline$a$ & -50.0 & -33.33 & -16.6 & 0.00 \\
$b$ & -50.0 & +11.11 & -15.3 & 1.68 \\
$c$ & -50.0 & +33.33 & -15.3 & 5.04 \\
$d$ & 0.0 & -33.33 & -4.3 & -10.08 \\
$e$ & 0.0 & +11.11 & -3.1 & +5.88 \\
$f$ & +50.0 & -33.33 & +5.5 & 0.00 \\
$g$ & +50.0 & +11.11 & +20.3 & 0.00 \\
$h$ & +50.0 & +33.33 & +28.8 & 0.00 \\
\hline \hline
\end{tabular}

We will use the crossing diagrams to evaluate the critical disorder strength and the critical conductivity for six more parameter sets $(U, n)$, which are listed in Table I. For every parameter set, the value of the critical dc conductivity is determined to an accuracy of $\sim 0.01 e^{2} / h$.

Table I lists four dimensionful quantities with different dimensions. $U$ and $\Delta_{\mathrm{c}}$ are in units of the hopping $t$, the filling $n$ is a dimensionless number, while $\sigma_{\mathrm{dc} \text {,crit }}$ is in units of the quantum of conductance, $e^{2} / h$. Thus, a direct comparison of the spread of these four quantities is meaningless. Therefore, we determine the relative spread of these four quantities across the different sets $a-h$. The reference point with respect to which the relative spread is calculated for the quantity of interest is taken to be the mean of the eight data sets. For example, the reference point for the Hubbard interaction $U$ is taken to be $\tilde{U}=\frac{1}{8}(1.0+1.0+$ $1.0+2.0+2.0+3.0+3.0+3.0)=2.0$. Note that $\tilde{U}$ has no physical meaning at this point and is simply taken to be the point of reference with respect to which relative spreads are calculated.

Table II shows the relative spreads of $U, n, \Delta_{\mathrm{c}}$, and $\sigma_{\mathrm{dc} \text {, crit }}$ for the sets $a-h$. The results collected in Table II can be summarized as follows: in spite of the strong variation of

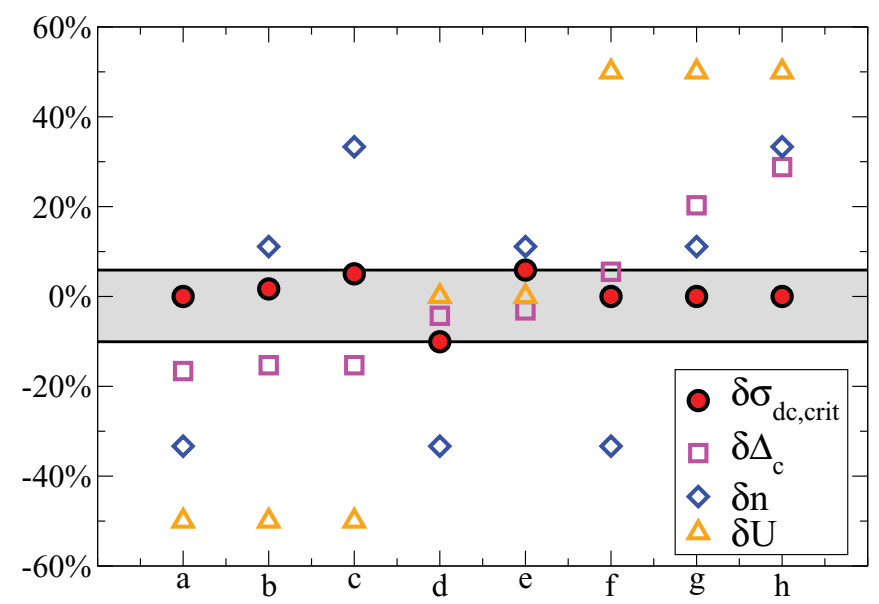

FIG. 5. (Color online) Graphical presentation of the spread of the parameters listed in Table II in percentage relative to $\tilde{p}$, where $p$ is one of the quantities $U, n, \Delta_{\mathrm{c}}$, or $\sigma_{\mathrm{dc}, \text { crit }}$. The gray band is a guide to the eye to emphasize the clustering of the values of $\sigma_{\mathrm{dc} \text {, crit }}$ compared to $U, n$, and $\Delta_{\mathrm{c}}$, not a mathematical measure of uncertainty. the microscopic input parameters $U, n$ and of the critical disorder strength $\Delta_{\mathrm{c}}$, the associated critical dc conductivity $\sigma_{\mathrm{dc} \text {,crit }}$ is found to be essentially independent of these input parameters. The results are presented graphically in Fig. 5, where the values of the critical conductivity are seen to cluster around the value $\sigma_{\mathrm{dc} \text {,crit }}=(1.19 \pm 0.02) e^{2} / h$, where the error $0.02 e^{2} / h$ is simply the error in estimating the arithmetic mean from the eight datasets. ${ }^{39}$ This provides evidence for the existence of a universal value of the critical conductivity. Indeed, the $\mathrm{NL} \sigma \mathrm{M}$, in which the dc conductivity appears as a coupling constant, predicts a universal ${ }^{40}$ critical value of the conductivity $\sigma_{\mathrm{dc} \text {, crit }} \sim 1.06 e^{2} / h,{ }^{19}$ in close correspondence with our result. In obtaining this estimate, we assumed that the number of valleys appropriate for our work is $n_{v}=1$. $^{41}$ Thus our results establish a link between the microscopic Anderson-Hubbard model and the low-energy effective theory provided by the NL $\sigma \mathrm{M}$ for the metal-insulator phase transition in two dimensions.

\section{CONCLUSIONS AND OUTLOOK}

The calculations presented in this paper were performed at finite temperature and for finite system sizes. While a quantum critical point (QCP) is defined at zero temperature and in the thermodynamic limit, it is well known to show its effects even at finite temperatures. ${ }^{18}$ Even if a spontaneous symmetry breaking unrelated to charge transport (perhaps magnetic in nature) were to completely mask the QCP between a metallic and an Anderson insulting phase, the existence of a universal conductivity at criticality can show up as one crosses the separatrix (by changing, in our case, disorder strength) between the metal and Anderson insulator at low but finite temperatures. We are currently investigating possible signatures of magnetic ordering in the Anderson-Hubbard model at even lower temperatures.

In summary, quantum Monte Carlo simulations of interacting lattice electrons in the presence of disorder for $d=2$ provide clear evidence for a transition from metallic to insulating behavior as the disorder strength is varied. At the transition, the value of the dc conductivity is found to be given by $\sigma_{\mathrm{dc} \text {, crit }}=(1.19 \pm 0.02) e^{2} / h$, implying that the critical dc conductivity is essentially independent of interaction strength, electron density, and the critical disorder strength. This points toward the existence of a universal critical dc conductivity. We obtained qualitatively similar results from investigations where site disorder is changed to bond disorder, the details of which will be given in a future presentation.

This work was supported in part by the SFB 484 (2000-2009) of the Deutsche Forschungsgemeinschaft and the TRR 80. One of us (KB) also acknowledges support through the grant N N202 103138 of the Polish Ministry of Science and Education. We are grateful for valuable discussions and communications with A. P. Kampf, S. M. Girvin, B. Altshuler, S. Sachdev, N. Trivedi, R. T. Scalettar, B. van Tiggelen, S. Chiesa, A. M. Finkel'stein, and J. Tworzydło. 
*prabuddha.chakraborty@physik.uni-augsburg.de

${ }^{1}$ P. A. Lee and T. V. Ramakrishnan, Rev. Mod. Phys. 57, 287 (1985).

${ }^{2}$ B. L. Altshuler and A. G. Aronov, in Electron-Electron Interactions in Disordered Systems, edited by M. Pollak and A. L. Efros (NorthHolland, Amsterdam, 1985), p. 1.

${ }^{3}$ D. Belitz and T. R. Kirkpatrick, Rev. Mod. Phys. 66, 261 (1994).

${ }^{4}$ E. Abrahams, S. V. Kravchenko, and M. P. Sarachik, Rev. Mod. Phys. 73, 251 (2001).

${ }^{5}$ S. V. Kravchenko and M. P. Sarachik, Rep. Prog. Phys. 67, 1 (2004). ${ }^{6}$ N. F. Mott, Metal-Insulator Transitions, 2nd ed. (Taylor and Francis, London 1990).

${ }^{7}$ P. W. Anderson, Phys. Rev. 109, 1492 (1958).

${ }^{8} 50$ Years of Anderson Localization, edited by E. Abrahams (World Scientific, Singapore, 2010).

${ }^{9}$ A. M. Finkelshtein, Sov. Phys. JETP 75, 97 (1983).

${ }^{10}$ C. Castellani, C. Di, P. A. Lee, and M. Ma, Phys. Rev. B 30, 527 (1984).

${ }^{11}$ M. A. Tusch and D. E. Logan, Phys. Rev. B 48, 14843 (1993); 51, 11940 (1995).

${ }^{12}$ D. L. Shepelyansky, Phys. Rev. Lett. 73, 2607 (1994).

${ }^{13}$ I. Bloch, J. Dalibard, and W. Zwerger, Rev. Mod. Phys. 80, 885 (2008); A. Aspect and M. Inguscio, Phys. Today 62, 30 (2009); L. Sanchez-Palencia and M. Lewenstein, Nature Phys. 6, 87 (2010).

${ }^{14}$ E. Abrahams, P. W. Anderson, D. C. Licciardello, and T. V. Ramakrishnan, Phys. Rev. Lett. 42, 673 (1979).

${ }^{15}$ L. P. Gor'kov, A. I. Larkin, and D. E. Khmel'nitskii, Zh. Eksp. Teor. Fiz., Pis'ma Red. 30, 248 (1979); JETP Lett. 30, 248 (1979).

${ }^{16}$ S. V. Kravchenko, G. V. Kravchenko, J. E. Furneaux, V. M. Pudalov, and M. D'Iorio, Phys. Rev. B 50, 8039 (1994).

${ }^{17}$ S. V. Kravchenko, W. E. Mason, G. E. Bowker, J. E. Furneaux, V. M. Pudalov, and M. D'Iorio, Phys. Rev. B 51, 7038 (1995).

${ }^{18}$ S. Sachdev, Quantum Phase Transitions (Cambridge University Press, Cambridge, 1999); H. v. Löhneysen, A. Rosch, M. Vojta, and P. Wölfle, Rev. Mod. Phys. 79, 1015 (2007).

${ }^{19}$ A. Punnoose and A. M. Finkel'stein, Science 310, 289 (2005).

${ }^{20}$ S. Anissimova, S. V. Kravchenko, A. Punnoose, A. M. Finkel'stein, and T. M. Klapwijk, Nature Phys. 3, 707 (2007).

${ }^{21}$ M. P. A. Fisher, G. Grinstein, and S. M. Girvin, Phys. Rev. Lett. 64, 587 (1990).

${ }^{22}$ A. M. Goldman and N. Marković, Phys. Today 51, 39 (1998).

${ }^{23}$ L. Schweitzer and P. Markos, Phys. Rev. Lett. 95, 256805 (2005).

${ }^{24}$ P. M. Ostrovsky, I. V. Gornyi, and A. D. Mirlin, Phys. Rev. Lett. 98, 256801 (2007).

${ }^{25}$ M. A. Tusch and D. E. Logan, Phys. Rev. B 48, 14843 (1993).

${ }^{26}$ D. Heidarian and N. Trivedi, Phys. Rev. Lett. 93, 126401 (2004).
${ }^{27}$ P. J. H. Denteneer, R. T. Scalettar, and N. Trivedi, Phys. Rev. Lett. 83, 4610 (1999); 87, 146401 (2001).

${ }^{28}$ P. J. H. Denteneer and R. T. Scalettar, Phys. Rev. Lett. 90, 246401 (2003).

${ }^{29}$ P. B. Chakraborty, P. J. H. Denteneer, and R. T. Scalettar, Phys. Rev. B 75, 125117 (2007).

${ }^{30}$ V. Dobrosavljević and G. Kotliar, Phys. Rev. Lett. 71, 3218 (1993).

${ }^{31}$ V. Dobrosavljević, A. A. Pastor, and B. K. Nikolić, Europhys. Lett. 62, 76 (2003).

${ }^{32}$ K. Byczuk, W. Hofstetter, and D. Vollhardt, Phys. Rev. Lett. 94, 056404 (2005); 102, 146403 (2009).

${ }^{33}$ D. Semmler, K. Byczuk, and W. Hofstetter, Phys. Rev. B 81, 115111 (2010).

${ }^{34}$ L. A. Tracy, E. H. Hwang, K. Eng, G. A. Ten, E. P. Nordberg, K. Childs, M. S. Carroll, M. P. Lilly, and S. Das Sarma, Phys. Rev. B 79, 235307 (2009).

${ }^{35}$ R. Blankenbecler, D. J. Scalapino, and R. L. Sugar, Phys. Rev. D 24, 2278 (1981).

${ }^{36}$ M. Suzuki, Prog. Theor. Phys. 56, 1454 (1976).

${ }^{37}$ J. E. Hirsch, Phys. Rev. B 31, 4403 (1985).

${ }^{38}$ M. Randeria, N. Trivedi, A. Moreo, and R. T. Scalettar, Phys. Rev. Lett. 69, 2001 (1992).

${ }^{39}$ The final error-value $\epsilon=0.02 e^{2} / h$ is obtained from the expression $\epsilon=\sqrt{\frac{1}{N(N-1)} \sum_{i=a}^{h}\left(x_{i}-x_{\mathrm{ave}}\right)^{2}}=\frac{s}{\sqrt{N-1}}$, where $x_{i}$ is the value of $\sigma_{\mathrm{dc} \text {, crit }}$ from the $i$ th parameter set, $x_{\mathrm{ave}}$ is the average of $\sigma_{\mathrm{dc} \text {, crit }}$ over the parameter sets $a-h, N=8$, and $s$ is the standard deviation of the critical dc-conductivity values across the datasets $a-h$. Even though some of the critical dc conductivities show deviation from the mean value, the overwhelming clustering of the dc-conductivity values around the mean value makes the error remarkably small.

${ }^{40}$ B. Spivak, S. V. Kravchenko, S. A. Kivelson, and X. P. A. Gao, Rev. Mod. Phys. 82, 1743 (2010).

${ }^{41}$ In Ref. [19], the authors define a dimensionless resistance $\rho=$ $\left[e^{2} /(\pi h)\right] R$ where $R$ is the dimensionful resistance. To include the number of valleys $n_{v}$ in the theory, a further dimensionless resistance $t$ is defined as $t=n_{v} \rho$. The renormalization group flow equations are derived by perturbatively expanding in a small parameter $1 / n_{v}$ around the limit $n_{v} \rightarrow \infty$. The authors find evidence for a quantum critical point between an Anderson insulator and a metallic state. At the critical point, the parameter $t$ takes the value $t_{C} \sim 0.3 .{ }^{19}$ To compare results obtained by us for the single-band Anderson-Hubbard model on a square lattice, we set $n_{v}=1$. It then follows that $\rho_{C}=t_{C} \sim 0.3$ at the critical point. Defining a critical conductivity $\sigma_{C}=1 / \rho_{C}$, this implies $\sigma_{C} \sim 1 / 0.3=3.33$ at the transition. In units of the unit of conductance $e^{2} / h$, we thus obtain $\sigma_{C} \sim 3.33\left[e^{2} /(\pi h)\right]=1.06\left(e^{2} / h\right)$. 